\title{
Wald und Waldnutzung im Umbruch des 18. Jahrhunderts
}

Das Aussehen und Gedeihen eines Waldes wird maßgeblich durch die spezifischen, sich ständig wandelnden Bedürfnisse des wirtschaftenden Menschen geprägt. Der Wald, der noch bis ins letzte Jahrhundert als Weide für allerlei Groß- und Kleinvieh diente und zugleich der Gewinnung von Bau-, Werk- und Brennholz genügen mußte, kann in seinem Aussehen unmöglich dem heutigen Idealbild eines vielfältig durchmischten, geschlossenen Hochwaldes entsprechen. Eine solche Erwartung läßt einen derart genutzten Weidewald als hoffnungslos übernutzt und degeneriert erscheinen. Die im Gegensatz zu heute völlig unterschiedlichen Funktionen eines Waldes führen dazu, daß es vor 1800 kaum eindeutige Grenzen zwischen Wald und übrigem Kulturland gab und eine Fixierung meist auch unnötig war; wo hörte der Wald auf, und wo fing das Allmendweideland an? Im extensiv bewirtschafteten Allmendland verliehen, je nach Geländeform, Hecken, Stauden, Einzelbäume, Baumgruppen und Gehölze der frühzeitlichen Landschaft ihr typisches Gepräge. 1764 protokollierte die Naturforschende Gesellschaft über die Allmend von Hausen am Albis: «Ihrer Beschreibung nach, sollte man vermuthen, ihr Gemeindewerk sehe einem Baumgarten gleich; In demselben hat ein jeder seine eigete und besondere Frucht-Bäume (Obstbäume)» (STAZ B IX 67, S. 43). Dörrfrüchte spielten damals besonders im Winter eine wichtige Rolle in der Ernährung der Menschen.

Die zwischenzeitliche Nutzung von Waldboden als Acker- und Getreideland dürfte in der überwiegend agrarisch geprägten Gesellschaft besonders in Notzeiten eine wichtige Bedeutung gehabt haben. Trotz Dreizelgenbrachwirtschaft gab es in der Allmend und im Wald einzelne Nischen, welche durchaus flexibel und individuellen Bedürfnissen entsprechend genutzt werden konnten. Die oftmals knapp an der Grenze zum Hungertod lebenden Menschen wußten die ausgleichende Wirkung des Waldes auf das Mikroklima und die erhöhte Fruchtbarkeit des Bodens durch Aschedüngung zu nutzen. Bereits gegen den Strom seiner Zeit schwamm der Historiker und Philosoph Ernst Moritz ARNDT, als er 1815 noch immer für eine gemischte Nutzungsform, besonders die Waldfeldwirtschaft, plädierte: «Was von Viehzucht und Ackerbau und Baumzucht in den Wäldern sich findet, ist mit Ausnahme der höchsten Gipfel, besonders gedeihlich, weil der Walddunst die Felder und Wiesen, welche zwischen liegen, vorzüglich die letzteren, außerordentlich fruchtbar macht» (zit. nach BARTHELMESS 1972, S. 63).
Heute gelten ganz selbstverständlich für die Bewirtschaftung des Waldes und diejenige des übrigen Kulturlandes verschiedene Gesetzesgrundlagen. Für die Verwaltung und Planung in der Forst- und Landwirtschaft sind unterschiedlich ausgebildete Fachleute zuständig, genauso wie die Arbeit im Wald professionalisiert und weitgehend abgekoppelt von derjenigen in der Landwirtschaft erfolgt. Diese Trennung unserer Kulturlandschaft in landwirtschaftliche und forstwirtschaftliche Zuständigkeitsbereiche gab es noch bis in die Zeit um 1800 nicht. Sie ist ein Produkt der frühen Neuzeit, wobei der allmähliche Ausdifferenzierungsprozeß in der zweiten Hälfte des 18. Jahrhunderts wesentliche Impulse erhielt. Erst grundlegende Neuerungen innerhalb der damaligen Landwirtschaft - in der Geschichte werden diese Veränderungen mit den Begriffen "Agrarmodernisierung» oder "Agrarrevolution" bezeichnet - ermöglichten die Schaffung eines eigenen Bereiches "Forstwirtschaft». Dieser Prozeß soll nachfolgend am Beispiel der Vorgänge in Stadt und Landschaft Zürich näher beleuchtet werden. Ein spezielles Augenmerk wird dabei auf jene Veränderungen gerichtet, welche mithalfen, den bäuerlich genutzten Weidewald zum «forstlich eingerichteten» Wirtschaftswald umzubauen.

Die Zürcher Bevölkerung hatte sich in den Jahren von 1700 bis 1792 trotz Hungerkrisen mehr als verdoppelt. Dieses Wachstum gründete im Zürcher Oberland weitgehend auf der Heimarbeit. Spinnen und Weben für das Baumwollgewerbe boten landarmen Bauern und landlosen Taunern oder Hintersassen, wie diese auch genannt wurden, eine willkommene Erwerbsmöglichkeit und garantierten letztlich ein Überleben. Die seit der Reformation nahezu unverändert gebliebene Agrarordnung vermochte die gestiegenen Nahrungsmittelbedürfnisse nicht mehr sicherzustellen. 20000 Menschen starben den Hungertod in den Jahren 1770/71, als lange, harte Winter die ohnehin von Mangel und Seuchen geschwächte Bevölkerung zusätzlich heimsuchten (GRABER 1991, S. 83/84).

\section{Dreizelgenbrachwirtschaft und Allmendnutzung}

BRAUN (1984) legte in seiner Studie "Das ausgehende Ancien Régime in der Schweiz» vergleichend dar, daß

Margrit Irniger, lic. phil. I, Historisches Seminar, Universität Zürich, Künstlergasse 16, 8006 Zürich 
zwischen dem markanten Bevölkerungswachstum, der Protoindustrialisierung und der Agrarordnung relevante Zusammenhänge bestehen. Dabei zeigte sich, daß gerade das fruchtbarste, von Topographie und Bodenqualität her privilegierte Gebiet des Zürcher Unterlandes, wo die Dreizelgenbrachwirtschaft dominierte, noch bis um 1800 wesentlich hinter dem Gesamtwachstum zurückblieb (BRAUN 1984, S. 33 ff.). Hemmnisse für die stagnierende oder gar rückgängige Agrarproduktion im 18. Jahrhundert bildeten die starke Belastung durch Zins- und Zehntenabgaben, weiter eine enorme Verschuldung, mit der fast alle Bauernhöfe und Gewerbebetriebe belastet waren, sowie Auswanderung und Abwanderung von Bauern in nichtagrarische Erwerbszweige (MEIER 1986, S. 240). Nebst den ohnehin limitierten Möglichkeiten der Ressourcenausschöpfung wird als Haupthindernis gemeinhin das enge Grenzen setzende System der Bodennutzung angesehen: Die Dreizelgenbrachwirtschaft mit ihrem Flurzwang, den vorgeschriebenen Anbauprodukten und -zeiten, mit Frühlings-, Herbst- und Brachweide sowie mit Zäunungsvorschriften engten den individuellen Spielraum eines Bauern oder Genossen beträchtlich ein.

In den meisten Dörfern der Zürcher Landschaft war das für die Getreideproduktion geeignete Land in drei große Zelgen aufgeteilt. Die erste Zelge wurde - im dreijährigen Anbaurhythmus - im Herbst mit Wintergetreide, meist Korn oder Roggen, die zweite im Frühling mit Sommergetreide, Gerste oder Hafer, angebaut, während die dritte brachlag und zusammen mit dem Allmendland der gemeinsamen Groß- und Kleinviehweide diente. Im folgenden Jahr wurde die Winterzelge brachgelegt, während Sommerzelge und Brache mit Getreide bebaut wurden. In jeder Zelge besaß der einzelne berechtigte Bauer eine Parzelle, deren Bewirtschaftung mangels Zufahrtswegen und im Verband mit den anderen Besitzern detailliert geregelt werden mußte. Nur die von Hof und Dorf weiter entfernten Landstücke, meist von minderer Bodenqualität, durften eingezäunt und für eine gewisse Zeit individuell genutzt werden.

Wälder, Weiden und wenig produktives Land (auch Moore, Flußauen oder Seeufer) außerhalb der Zelgen wurden von den Dorfgenossenschaften gemeinsam als Allmend genutzt. Die kollektive Nutzungsberechtigung war an Hof- und Grundbesitz geknüpft und nicht an Personen. Die Zahl dieser sogenannten Gerechtigkeiten war zwar fixiert, doch fanden Aufteilungen bei Vererbungen oder Verkauf statt, was bereits im Spätmittelalter zu einer Versplitterung der Rechte geführt hatte. Großbauern mit viel Vieh profitierten mehr von der gemeinsamen Allmendweide, während die kleinbäuerlichen Tauner mit ihrer Kuh oder den zwei Ziegen oftmals nur auf Zusehen hin mitnutzen durften. Diese an Besitz gebundene Allmendnutzungsberechtigung barg so wesentlichen sozialen Konfliktstoff in sich.

Die privilegierten Inhaber einer solchen Gerechtigkeit besaßen üblicherweise nebst dem kollektiven Weiderecht auch das Recht, im jährlich von der Obrigkeit zur Nutzung bezeichneten Holzhau (Schläge) Brenn- und
Zaunholz für den Eigenbedarf zu schlagen. Bauholz für einen Neubau oder für die Reparatur von Wohn- und Wirtschaftsgebäuden sowie $\mathrm{Holz}$ für Geräte wurden meist nur auf Gesuch hin zugestanden. Auch hier profitierten Großbauern mit ihrem höheren Eigenbedarf an Holz am meisten. Generell diente die Allmend nicht nur als Weideland und Holzlieferant, sondern war auch - je nach den naturräumlichen Gegebenheiten - Lieferant von Riedstreue und von sämtlichen irgendwie verwertbaren, natürlich wachsenden Waldpflanzen wie Pilzen, Beeren oder Heilkräutern.

\section{Klagen über Holznot}

Knapper werdende Ressourcen waren spätestens seit dem 16. Jahrhundert handlungsleitend bei der Regelung der dörflichen Allmendweide- und Holznutzung. Die zahlenmäßig weit überwiegende, ländliche Bevölkerung, die immer wieder mit Ernteausfällen, Hungersnöten, Seuchen und Krieg konfrontiert war, wußte genau um die Endlichkeit des Rohstoffes Holz. Innerhalb des Dorfes sollten genaue Regelungen des Weidgangs, der Holzausgaben, des Schutzes von Jungwüchsen sowie sämtlicher Nutzungen in Wald und Feld Konflikte im Umgang mit den Ressourcen vermeiden helfen. Das labile Gleichgewicht zwischen traditioneller, bäuerlicher Waldnutzung und der verstärkt auf den alleinigen Holznutzen bedachten Interessen der städtischen Obrigkeit, angesichts einer steigenden Nachfrage nach Holz, drohte immer wieder zu kippen.

Mit Klagen über Holzmangel und -teuerung beschäftigten sich wiederholt Holzkommissionen, die aus Mitgliedern des Rats zusammengesetzt waren. In einem Bericht von 1763 erklärte die damalige Holzkommission die Ursachen des erneuten Holzmangels primär mit der höheren Gewalt der Natur: Zu niedriger Wasserstand der Sihl verhinderte ein Heranflößen von Holz aus dem Sihlwald und dem Gebiet um Einsiedeln. Andererseits zerstörten Hochwasser die Wehre und schwemmten das Flößholz die Limmat hinunter. Die notwendigen Reparaturen zur Flößbarmachung der Sihl, dem für die Stadt Zürich zentralen Holzzubringersystem, verschlangen selber enorme Holzmengen. Auch Schneemangel im Winter verunmöglichte das Heranschlitten von Holz. Weiter bemängelten die Kommissionsmitglieder in ihrer Expertise das eigennützige Verhalten reicher Bürger, welche große Holzvorräte horteten, während sich Arme auf dem Markt kaum mit dem nötigsten Brennholz versorgen könnten. Als Preistreiber gerieten die Holzschiffleute und -händler unter Beschuß, da sie das Holz bis in den Winter zurückbehielten und erst nach Ansteigen der Preise auf den Markt brächten. Auch wurde immer wieder qualitativ minderes Holz («halb faules, im Wasser verblagtes») unter dem guten versteckt und zu überhöhten Preisen abgesetzt. Zur Abhilfe der Holzknappheit empfahl die Kommission schließlich vermehrte Holzimporte, wobei zögernde Handelspartner nötigenfalls mit Vorauszahlungen zum definitiven Vertragsabschluß 
ermuntert werden sollten (STAZ A 65.4, 13.5.1763; allg. vgl. RADKAU/SCHÄFER 1987, S. 101 u. 150 ff.).

In der ersten Hälfte des 18. Jahrhunderts dominierten als obrigkeitliche Maßnahmen, die gegen eine zwar drohende Holzknappheit ergriffen wurden, noch traditionelle Mittel: Mit dem Erlaß von Wald- und Holzordnungen oder Mandaten regelte der Zürcher Rat vor allem die herkömmlichen, bäuerlichen Holznutzungs- und Waldweiderechte immer restriktiver und bis ins Detail. Sich häufende Streitigkeiten im 16. Jahrhundert um Nutzungsanteile in der Allmend hatten vielenorts zum Ausschluß von Hintersassen geführt ebenso wie von Hofbebauern, welche außerhalb des Dorfetters siedelten. Mit hohen Einzugsgeldern wurden mittellose Zuwanderer vom Dorf, und damit von der Mitnutzung der Allmend, ferngehalten. Auch waren die Holzbezüge schon früh quantitativ wie qualitativ festgesetzt worden. Die Kontrolle darüber, welches Holz wo, wann und von wem genutzt werden durfte, war wesentlich verschärft worden (IRNIGER 1991, S. 77 ff.).

Die Mandate und Holzordnungen wurden seit dem Spätmittelalter regelmäßig mit drohendem Holzmangel, mit völlig ausgeholzten und überweideten Wäldern begründet und die Gemeinden zum Sparen angehalten. Die zur formelhaften Wendung erstarrte Einleitung der jeweiligen Ordnung fordert eine quellenkritische Skepsis geradezu heraus. Der angebliche Schutz, die vermehrte Schonung der Wälder durch den Erlaß von Mandaten schließt die Interessengebundenheit einer geregelten Holznutzung keineswegs aus. Unter dem Vorwand von drohendem Holzmangel demonstrierte der Landesherr mit neuen Verordnungen oftmals seine Herrschaftsansprüche und kaschierte steuerliche oder territoriale Interessen (vgl. dazu die Studien von ALLMANN 1989 und SCHÄFER 1992).

\section{Steinkohle und Torf als Brennholzersatz}

Was dagegen seit Beginn des 18. Jahrhunderts neu in Angriff genommen und vom städtischen Rat finanziell und ideell stark gefördert wurde, war die Beschaffung einer alternativen Energiequelle. Steinkohle und Torf hatten als neuentdeckte Brennstoffe vermehrt Holz und Holzkohle zu ersetzen. Brennholz konnte so gespart, die Holzpreise konnten gesenkt sowie die Wälder geschont werden. Von Gelehrten und Beamten waren die neuen Brennstoffe geradezu euphorisch begrüßt worden, hieß es doch beim Kameralisten Johann Friedrich PFEIFFER 1775 in seiner gedruckten "Geschichte der Steinkohlen und des Torfs", daß Industrialisierung und Bevölkerungswachstum in Holland und England ohne diese Rohstoffe gar nie in dieser Weise hätte stattfinden können, «wenn die allzeit beschäftigte Natur nicht andere Brennmittel in ihrer unterirdischen Vorrathskammer fabriciert, und die Noth diese Nationen nicht gelehrt hätte, selbige aufzusuchen, und anzuwenden" (S. 4). Weiter schrieb PFEIFFER (1775, S. 5): «Die freygebige Natur hatte in ihrer unterirdischen Werkstatt so fleißig gearbeitet, ja so mütterlich gesorget, dass man nicht lange suchen durfte.» Deutlich zeigt sich hier der Glaube an die Unerschöpflichkeit der Natur, der Glaube auch an den technischen Fortschritt, der es dem Menschen erlaubt, sich bisher unbekannte Ressourcen nutzbar zu machen.

Der gleiche Autor beschäftigte sich in seinem «Grundris der Forstwissenschaft» ausführlich mit "Holzsparkünsten", wo er einführend bemerkte, daß sich die Staaten zuwenig um den Holzverbrauch kümmern würden und die "Holzverschwendung» besonders in der Schweiz «maßlos» sei mit ihren "Kachelöfen von außerordentlicher Größe» (PFEIFFER 1781, S. 318/19). Der Vorwurf mangelnder Holzsparsamkeit traf auch Zürcher Bürger, als die Holz- oder Waldungskommission in ihrem Bericht 1763 «überhandnehmenden Luxe und Weichlichkeit» feststellte, begleitet von vermehrtem "Consum» und einer "Sorglose(n) Vergeüdung und Verschwendung» von Holz (STAZ A 65.4). Schuldzuweisungen und Raubbauvorwürfe gehörten im 18. Jahrhundert geradezu zur Tagesordnung und dienten oftmals dazu, die Lage noch schwärzer zu malen, als sie schon war. Dadurch sollte das Klima für die angestrebten Neuerungen - die Verbesserung der Landwirtschaft und der Waldungen, Verwendung von Steinkohle oder Torf - allmählich geschaffen werden.

\section{Zürcher Ökonomen und Agrarmodernisierung}

Die 1759 gegründete Ökonomische Kommission, ein Fachausschuß der Physikalischen oder später Naturforschenden Gesellschaft, wurde im zürcherischen Gebiet zur treibenden Kraft der Agrarreformen. Ihre Mitglieder stammten durchwegs aus gutgestellten, reichen und regierungsbeteiligten Zürcher Bürgergeschlechtern. Für die Probleme der Landbevölkerung waren sie durch drastische Hunger- und Elendsschilderungen mehr und mehr sensibilisiert worden. Außerdem waren sie in ihrem Interesse für die ländliche Bevölkerung und deren Lebensgrundlage - die Landwirtschaft - dem weitaus wichtigsten Sektor der damaligen Volkswirtschaft, wesentlich beeinflußt von englischen und französischen ökonomischen Gesellschaften. Der Zustand der Landwirtschaft oder "Land Öconomie», wie es damals noch hieß, in den einzelnen Gemeinden sollte systematisch erhoben und tabellarisch erfaßt werden, einerseits, um die Mängel herauszufinden, und andererseits, um die Produktion zu erhöhen und damit die Nahrungsgrundlage zu erweitern (GRABER 1991, S. 85/86). Das erklärte Ziel, die Ertragssteigerung in der Landwirtschaft, erforderte durch die personell, räumlich-organisatorisch und rechtlich eng verflochtenen Bodennutzungsformen auch die Berücksichtigung von Wald und Allmend. Die in der Stadt Zürich immer wieder ertönenden Holzmangelklagen und deren Behandlung durch Holzkommissionen hatten die problematischen Berührungspunkte zwischen den herkömmlichen Ackerbau-, Viehwirtschafts- und Waldnutzungsformen bereits zur Genüge bewußt gemacht. 
So war es naheliegend, daß die Zürcher Ökonomen in ihrer praktischen Tätigkeit an den Vorarbeiten der Holzkommissionen anknüpften. Umgekehrt ließ sich diese jetzt als Waldungs- und Forstkommission bezeichnet beim Ausarbeiten einer neuen, 1773 vom Rat verabschiedeten Forstordnung (Erneuerung des "Waldungsmandates» von 1711 und 1717) stark von den Ideen der Ökonomen beeinflussen (KREBS, SCHULER und WITSCHI 1983 S. 34 ff.).

Mittels Preisausschreibungen und sogenannten Bauerngesprächen suchten sie Kontakte zu einzelnen Gemeinden und vor allem zu den praktischen Erfahrungen der Landleute. Praktische Kenntnisse und das Wissen über neue, vorwiegend technische Verfahren sollten bei dieser Gelegenheit ausgetauscht und die Landleute zu Innovationen ermuntert werden. Grundlage für diese Gespräche und allgemein für Kosten-Nutzen-Rechnungen der bäuerlichen Wirtschaft bildeten die sozialen und wirtschaftlichen Daten, die die Ökonomen auf der Landschaft sammelten. Als konkrete Maßnahmen zur Verbesserung der Landwirtschaft propagierten die Ökonomen besonders die neue Anbaumethode der Fruchtwechselwirtschaft mit vermehrter Düngerverwendung und dem Ansäen der Brachfelder mit Klee und anderen stickstoffreichen Futterpflanzen, sodann die Stallfütterung sowie die Aufteilung der Allmenden (BRAUN 1984, S. $100 \mathrm{ff}$; GRABER 1991, S. 87). Der anfallende Dünger bei Stallfütterung konnte viel gezielter verwendet, Weideflächen konnten umgenutzt und der Zaunholzverbrauch gesenkt werden. Allmendteilungen sollten die individuelle Verfügung über zusätzliches Kulturland bringen und dadurch Ansporn für eine intensivere Nutzung sein.

\section{Zürcher Ökonomen und Holzertragssteigerung}

Schon kurz nach der Gründung befaßten sich die Ökonomen intensiv mit vorwiegend waldnutzungstechnischen Fragen. Heinrich Götschi, Bannwart und Küfer von Oberrrieden, spielte hier eine ähnliche Rolle wie Kleinjogg, der Musterbauer und eifrige Experimentierer auf dem Gebiete der landwirtschaftlichen Ertragssteigerung. Götschi begann, angespornt von den Preisausschreibungen der Ökonomen, mit dem probeweisen Ansäen und Anpflanzen besonders von Nadelhölzern. Er berechnete, daß der Holzertrag eines Nadelwaldes denjenigen eines Laubwaldes bei weitem übersteige (STAZ B IX 67, S. 118). 1787 erhielt Bannwart Hotz, ein anderer Amtmann und Forstpraktiker der Stadt Zürich, auf eigenes Ersuchen hin einen zinslosen Kredit zur Einrichtung eines Magazins von Lärchen-, Föhren-, Rot- und Weißtannensamen. Er erhielt auch zugleich das Monopol für den Vertrieb, denn Zürichs Ämter und Vogteien wurden verpflichtet, den erforderlichen Samen bei ihm zu fixen Preisen zu kaufen (StZ III B 968, Nr. 164).

Alle Faktoren, die eine Aufforstung der Wälder beeinträchtigten, wurden nun genau und mit neuer Sensibilität beobachtet. 1793 meldete Hotz dem Zürcher Rechenrat pflichtschuldig: Fünf Rottannen im Neuforst seien durch «die verderbliche Einsäckt des Baum Käfer sehr stark angestekt gewäsen», so daß er die befallenen Stämme mitsamt Wurzeln, Rinde und Ästen aus dem Wald geschafft habe. Die Zürcher bestätigten ihm, daß dieses Vorgehen aufgrund früherer Erfahrungen das einzige Mittel zur Bekämpfung des Borkenkäfers sei (StZ III B 968, Nr.191). Heinrich Götschi war bemüht, sämtliche «öden» und wenig bestockten Plätze im Forst des Fraumünsteramtes mit möglichst schnell wachsenden Bäumen, vorwiegend Fichten und Lärchen, zu bepflanzen und so den Waldboden «fruchtbar zu machen». Dem Zürcher Rat versicherte er schon 1767: «Das Werk der Verbesserung der Waldungen ist nur zimlich schwehr, bis einmahl die durch Unerkentnis verdorbenen und versaumten Pläze nachgenommen und vervollkomnet sind, hernach ist es ein geringes, die Waldungen in einem guten Stand zu erhalten» (STAZ B IX 67, S. 117).

Einige Mitglieder der Ökonomen, unter ihnen etwa Johann Jakob Ott, der erste Präsident, bemühten sich beim Zürcher Rat um die Bewilligung einer forstlichen Versuchsfläche. Mit dem Sihlhölzli erhielten sie 1767 ein Grundstück für Pflanzungsversuche und zur Anlage «eines Lustwäldleins» und «Spaziergangs». Die Musteranlage sollte also mit einem parkähnlichen Charakter Erholungs- und Rückzugsort für Stadtmüde werden. Neben ästhetischen galten aber auch rationelle Ansprüche: Mit Trauerweide, Robinie - sogenannten Exoten - wurden erste Anpflanzungsversuche gemacht und überhaupt das Gedeihen der einzelnen Baumarten wie Pappeln, Maulbeerbäume (für die Zucht von Seidenraupen), Kastanien, Föhren, Lärchen, Tannen, Eichen und Buchen genau überwacht. Die verschiedenen Baumarten sollten in ihrer gesamten Wüchsigkeit miteinander verglichen werden (HAUSER 1963).

\section{Einrichtung der Forstwirtschaft}

Die Reformbestrebungen in der zweiten Hälfte des 18. Jahrhunderts ebneten den Weg für einschneidende Veränderungen bei der Waldnutzung und Forstorganisation, wie sie dann im Verlaufe des letzten Jahrhunderts etabliert und gesetzlich verankert werden konnten. Indem nämlich das Vieh gemäß den Empfehlungen der Ökonomen zunehmend im Stall gefüttert wurde, schwächte sich der Widerstand vieler Bauern gegen Einschränkungen der Waldweide und schließlich auch deren Verbot. Die Aufteilung der Allmenden, die Definition der Wälder als eindeutig abgegrenztes Grundeigentum und damit verbunden die Ablösung der Holznutzungsrechte (BRAUN 1984, S. 96 ff.) ermöglichten erst die Etablierung einer von landwirtschaftlichen Ansprüchen weitgehend losgelösten Forstwirtschaft. Die forstlichen Zielsetzungen waren auf einen reinen Nutzholzwald ausgerichtet. Die nötigen Schritte in diese Richtung unternahmen Forstpraktiker wie Götschi oder Hotz, Exponenten der Waldungskommissionen und der Ökonomen, welche die nötigen Verbindungen zur Zürcher Regierung besaßen oder mit der Zeit selber im Rat saßen. 
Das Werk einer Steigerung der Holzerträge wurde begonnen, indem zuerst einmal der Umfang und Zustand der Waldungen erfaßt werden mußte. Diese Arbeiten waren von der Waldungskommission für die obrigkeitlichen Waldungen bereits 1760 in Angriff genommen und tabellarisch dargestellt worden (KREBS, SCHULER und WITSCHI 1983, S. 32/33). Zweitens wurde mit Ansaaten und Bepflanzung von feuchten und wenig oder unbestockten Waldlichtungen begonnen, was meist eine vorgängige Bodenmelioration durch Anlegen von Entwässerungsgräben erforderte. Drittens wurden mit importierten, besonders schnellwüchsigen Baumarten wie Lärchen, Douglas oder Akazien Versuche gemacht und mit dem Gedeihen standortheimischer Baumarten verglichen. Die Auswahl der Baumarten orientierte sich zwar auch an den natürlichen Standortfaktoren, doch wurde der Geschwindigkeit des Wachstums Priorität eingeräumt. Der zum Aufforsten vorgesehene Waldboden wurde vergleichbar mit dem übrigen Kulturland als Ackerfläche betrachtet, hieß es doch in den Empfehlungen der Waldungskommission: «... wäre es von unbeschreiblichem Nutzen wegen geschwinder Vermehrung des Holzes, wann die Plätze, die ausgeholzt werden, auch alsobald darauf ausgestoket, umgepflüget, und mit Holzsamen dichte besäet würden" (STAZ A 64.4, 1763).

Besonderen Wert legten die ersten Forstleute auch auf eine «forstliche Einrichtung» der Wälder, denn diese ermöglichte erst die Berechnung der Nachhaltigkeit, d. h. das Prinzip, jährlich nicht mehr Holz zu nutzen, als im gleichen Zeitraum wieder nachwachsen kann. Zu diesem Zweck mußte der Wald vermessen und Pläne gezeichnet werden. 1792 erstellte Heinrich Hotz (der Sohn von Bannwart und Forstmeister Rudolf Hotz) einen ersten «Grundriß» vom Schneitenberg-Wald der Landvogtei Andelfingen. Dabei wurde das gesamte Waldareal auf dem Reißbrett in verschiedene Bezirke unterteilt, welche ihrerseits wieder in geometrisch exakte, fortlaufend numerierte Schlagflächen eingeteilt wurden (vgl. Abb. KREBS, SCHULER und WITSCHI 1983, S. 119). Solche Pläne wurden für die Festlegung der jährlichen Holznutzungsmenge zu einem wichtigen forstplanerischen Instrument, welches wesentlich verfeinert in Form von Wirtschaftsplänen noch heute diese Funktion hat.

\section{Kurzfassung}

In der zweiten Hälfte des 18. Jahrhunderts kamen im Hinblick auf den Wald wesentliche Veränderungen in Gang: Auf Initiative der Ökonomischen Kommission sollten die landwirtschaftlichen Erträge allmählich erhöht werden. Propagiert wurde deshalb der Übergang zur Fruchtwechselwirtschaft, die vermehrte Stallfütterung und Düngerverwendung sowie die Aufteilung der Allmenden. Solche Umstrukturierungen im Agrarbereich ermöglichten auch eine Neuorganisation der Waldnutzung. Befreit von überwiegend landwirtschaftlichen Nutzungsansprüchen, besonders der Waldweide, konnte die Funktion des Waldes neu definiert und primär auf den Holznutzen ausgerichtet werden. Mit der forstlichen Planung hielten auch Maßnahmen wie künstliche Ansaaten auf verlichteten Stellen, Anbauversuche mit neueingeführten Baumarten, Bodenmeliorationen, die Vermessung und Erstellung von Plänen und Tabellen ihren Einzug in der sich etablierenden Forstwirtschaft.

\section{Quellen}

Staatsarchiv Zürich (STAZ)

A 65.4 Akten Sihlamt, 1700-1798

B IX 67 Protokolle der Naturf. Gesellschaft, ab 1763

Stadtarchiv Zürich (StZ)

III B 968 Akten Forst, 1541-1795

PFEIFFER, J. F. (1775): Geschichte der Steinkohlen und des Torfs. Mannheim.

PFEIFFER, J.F. (1781): Grundriß der Forstwirtschaft, zum Gebrauche dirigierender Forst- und Kameralbedienten, auch Privatguthsbesitzern. Mannheim.

\section{Literatur}

ALLMANN, J. (1989): Der Wald in der frühen Neuzeit. Eine mentalitäts- und sozialgeschichtliche Untersuchung am Beispiel des Pfälzer Raumes 1500-1800 (= Schriften zur Wirtschafts- und Sozialgeschichte, 36), Duncker \& Humblot, Berlin.

BARTHELMESS, A. (1972): Wald. Umwelt des Menschen Dokumente zu einer Problemgeschichte von Naturschutz, Landschaftspflege und Humanökologie. Alber, Freiburg und München.

BRAUN, R. (1984): Das ausgehende Ancien Régime in der Schweiz. Vandenhoeck \& Ruprecht, Göttingen und Zürich.

GRABER,R. (1991): Spätabsolutistisches Krisenmanagement. Die Naturforschende Gesellschaft in Zürich im Spannungsfeld von arbeitender Geselligkeit und staatlicher Funktionalisierung. In: JOST und TANNER (Hrsg.), Geselligkeit, Sozietäten und Vereine (= Schweiz. Ges. f. Wirtschafts- und Sozialgeschichte, 9), Chronos, Zürich, S. 81-94.

HAUSER, A. (1963): Der erste schweizerische Versuchswald im Zürcher Sihlhölzli 1768-1808. In: Schweizerische Z.f. Forstwesen, 114, 505-516.

IRNIGER, M. (1991): Der Sihlwald und sein Umland. Waldnutzung, Viehzucht und Ackerbau im Albisgebiet von 1400-1600 (= Mitteilungen der Antiquarischen Gesellschaft in Zürich, 58), Verlag Hans Rohr, Zürich.

KREBS, E., SCHULER, A., WITSCHI, P. (1983): 650 Jahre zürcherische Forstgeschichte, Bd.1, Forstpolitik, Waldbenutzung und Holzversorgung im alten Zürich. Hg. v. Regierungsrat des Kantons Zürich und Stadtrat von Zürich, Zürich.

MEIER, T. (1986): Handwerk, Hauswerk, Heimarbeit. Nichtagrarische Tätigkeiten und Erwerbsformen in einem traditionellen Ackerbaugebiet des 18. Jh. (Zürcher Unterland). Chronos, Zürich.

RADKAU, J., SCHÄFER, I. (1987): Holz. Ein Naturstoff in der Technikgeschichte. Rowohlt, Rororo Sachbuch, Hamburg.

SCHÄFER, I. (1992): "Ein Gespenst geht um.” Politik mit der Holznot in Lippe 1750-1850 (= Sonderveröffentlichungen des Naturwissenschaftlichen Vereins Lippe, 38), Detmold. 